\title{
INTERACTION OF MASSIVE BLACK HOLE BINARIES WITH THEIR STELLAR ENVIRONMENT. II. LOSS CONE DEPLETION AND BINARY ORBITAL DECAY
}

\author{
Alberto Sesana, ${ }^{1}$ Francesco Haardt, ${ }^{1}$ and Piero Madau ${ }^{2,3}$ \\ Received 2006 December 8; accepted 2007 January 18
}

\begin{abstract}
We study the long-term evolution of massive black hole binaries (MBHBs) at the centers of galaxies using detailed scattering experiments to solve the full three-body problem. Ambient stars drawn from an isotropic Maxwellian distribution unbound to the binary are ejected by the gravitational slingshot. We construct a minimal, hybrid model for the depletion of the loss cone and the orbital decay of the binary and show that secondary slingshots - stars returning on small-impact parameter orbits to have a second superelastic scattering with the MBHB — may considerably help the shrinking of the pair in the case of large binary mass ratios. In the absence of loss cone refilling by two-body relaxation or other processes, the mass ejected before the stalling of a MBHB is half the binary reduced mass. About $50 \%$ of the ejected stars are expelled in a "burst" lasting $\sim 10^{4} \mathrm{yr} M_{6}^{1 / 4}$, where $M_{6}$ is the binary mass in units of $10^{6} M_{\odot}$. The loss cone is completely emptied in a few bulge crossing timescales, $\sim 10^{7} \mathrm{yr} M_{6}^{1 / 4}$. Even in the absence of two-body relaxation or gas dynamical processes, unequal mass and/or eccentric binaries with $M_{6} \gtrsim 0.1$ can shrink to the gravitational wave emission regime in less than a Hubble time and are therefore "safe" targets for the planned Laser Interferometer Space Antenna.
\end{abstract}

Subject headings: black hole physics — methods: numerical — stellar dynamics

Online material: color figure

\section{INTRODUCTION}

It is now widely accepted that the formation and evolution of galaxies and massive black holes (MBHs) are strongly linked; MBHs are ubiquitous in the nuclei of nearby galaxies, and a tight correlation is observed between hole mass and the stellar mass of the surrounding spheroid or bulge (e.g., Magorrian et al. 1998; Gebhardt et al. 2000; Ferrarese \& Merritt 2000; Haring \& Rix 2004). If MBHs were also common in the past (as implied by the notion that distant galaxies harbor active nuclei for a short period of their life), and if their host galaxies experience multiple mergers during their lifetime, as dictated by cold dark matter (CDM) hierarchical cosmologies, then close $\mathrm{MBH}$ binaries (MBHBs) will inevitably form in large numbers during cosmic history (Begelman et al. 1980). Observations with the Chandra satellite have indeed revealed two active MBHs in the nucleus of NGC 6240 (Komossa et al. 2003), and a MBHB is inferred in the radio core of $3 \mathrm{C} 66 \mathrm{~B}$ (Sudou et al. 2003). The Very Long Baseline Array (VLBA) discovery in the radio galaxy $0402+379$ of a MBHB system with a projected separation of just $7.3 \mathrm{pc}$ has recently been reported by Rodriguez et al. (2006). MBH pairs that are able to coalesce in less than a Hubble time will give rise to the loudest gravitational wave (GW) events in the universe. In particular, a low-frequency space interferometer like the planned Laser Interferometer Space Antenna (LISA) is expected to have the sensitivity to detect nearly all MBHBs in the mass range $10^{4}-10^{7} M_{\odot}$ that happen to merge at any redshift during the mission operation phase (Sesana et al. 2005). The coalescence rate of such "LISA MBHBs" depends, however, on the efficiency with which stellar and gas dynamical processes can drive wide pairs to the GW emission stage.

Following the merger of two halo $+\mathrm{MBH}$ systems of comparable mass ("major mergers"), it is understood that dynamical

\footnotetext{
1 Dipartimento di Fisica e Matematica, Universitá dell'Insubria, 22100 Como, Italy.

${ }^{2}$ Department of Astronomy \& Astrophysics, University of California, Santa Cruz, CA 95064.

3 Max-Planck-Institut für Astrophysik, 85740 Garching bei Muenchen, Germany.
}

friction will drag in the satellite halo (and its $\mathrm{MBH}$ ) toward the center of the more massive progenitor (see, e.g., Kazantzidis et al. 2005). This will lead to the formation of a bound MBH binary in the violently relaxed core of the newly merged stellar system. As the binary separation decays, the effectiveness of dynamical friction slowly declines, because distant stars perturb the binary's center of mass but not its semimajor axis (Begelman et al. 1980). The bound pair then hardens by capturing stars passing in its immediate vicinity and ejecting them at much higher velocities (gravitational slingshot). It is this phase that is considered the bottleneck of a MBHB's path to coalescence, as there is a finite supply of stars on intersecting orbits and the binary may be "hung up" before the back-reaction from GW emission becomes important. This has become known as the "final parsec problem" (Milosavljevic \& Merritt 2003, hereafter MM03).

While the final approach to coalescence of binary MBHs is still not well understood, several computational tools have been developed to tackle the problem at hand. The orbital decay rate depends on several parameters of the guest binary (mass, mass ratio, orbital separation, and eccentricity) and on the stellar distribution function of the host galaxy bulge. In early treatments (e.g., Mikkola \& Valtonen 1992; Quinlan 1996, hereafter Q96), the stellar ejection rate and the rate of change of the binary semimajor axis and eccentricity were derived via three-body scattering experiments in a fixed stellar background. The assumption of a fixed background breaks down once the binary has ejected most of the stars on intersecting orbits, and the extraction of energy and angular momentum from the binary can continue only if new stars can diffuse into low-angular momentum orbits (refilling the binary's phase-space "loss cone") or via gas processes (Escala et al. 2004; Dotti et al. 2006). Hybrid approaches in which the rate coefficients derived from numerical experiments in a fixed background are coupled with a model for loss cone repopulation have been used, e.g., by Yu (2002) and MM03, while the limiting case in which the loss cone is constantly refilled but the central stellar density decreases due to mass ejection has been studied in a cosmological context by Volonteri et al. (2003a, 2003b). A fully 
self-consistent, $N$-body approach to the evolution of MBHBs, while clearly desirable, is limited today to $N \lesssim 10^{6}$ particles, corresponding to a mass resolution of $m_{*} / M \sim 10^{-3}$ (e.g., Quinlan \& Hernquist 1997; Milosavljevic \& Merritt 2001; Hemsendorf et al. 2002; Aarseth 2003; Chatterjee et al. 2003; Makino \& Funato 2004; Berczik et al. 2005). Such performance figures are not sufficient to reproduce central bulges, even of faint galaxies; and the small particle numbers cause an artificial enhancement of starstar scatterings and of the Brownian motion of the binary, leading to a spurious refilling of the loss cone.

This is the second paper in a series aimed at a detailed study of the interaction of MBHBs with their stellar environment. In Sesana et al. (2006, hereafter Paper I), three-body scattering experiments were performed to study the ejection of hypervelocity stars (HVSs) by MBHBs in a fixed stellar background. In this paper we use a hybrid approach to investigate the orbital decay and shrinking of MBHBs in time-evolving stellar cusps. Numerically derived rates of stellar ejections stars are coupled to an extension of the analytical formulation of loss cone dynamics given by MM03. This method allows us to simultaneously follow the orbital decay of the pair as well as the time evolution of the stellar distribution function. MBHBs are embedded in the deep potential wells of galaxy bulges, so when the binary first becomes "hard" only a few stars acquire a kick velocity large enough to escape the host. The bulge behaves as a collisionless system, and many ejected stars will return to the central region on nearly unperturbed, small-impact parameter orbits and will undergo a second superelastic scattering with the binary, as first discussed by MM03. Under the assumption of a spherical potential, we quantify the role of these "secondary slingshots" in determining the hardening of the pair. The plan of the paper is as follows. In $\S 2$ we describe our hybrid model for the orbital evolution of MBHBs in a time-evolving stellar density profile. The shrinking and coalescence of the binary is discussed in $\S 3$.

\section{HARDENING IN A TIME-EVOLVING BACKGROUND}

\subsection{Scattering Experiments}

Our hybrid method relies on the large number of outputs from the suite of three-body scattering experiments presented in Paper I. In the following we briefly summarize the basic theory. Consider a binary of mass $M=M_{1}+M_{2}=M_{1}(1+q)\left(M_{2} \leq M_{1}\right)$, reduced mass $\mu=M_{1} M_{2} / M$, and semimajor axis $a$ orbiting in a background of stars of mass $m_{*}$. In the case of a light intruder with $m_{*} \ll M_{2}$, the problem is greatly simplified by setting the center of mass of the binary at rest at the origin of the coordinate system. It is then convenient to define an approximate dimensionless energy change $C$ and angular momentum change $B$ in a single binarystar interaction as (Hills 1983)

$$
\begin{gathered}
C=\frac{M}{2 m_{*}} \frac{\Delta E}{E}=\frac{a \Delta E_{*}}{G \mu}, \\
B=-\frac{M}{m_{*}} \frac{\Delta L_{z}}{L_{z}}=\frac{M}{\mu} \frac{\Delta L_{z *}}{L_{z}},
\end{gathered}
$$

where $\Delta E / E$ is the fractional increase (decrease if negative) in the orbital specific binding energy $E=-G M /(2 a), \Delta L_{z} / L_{z}$ is the fractional change in orbital specific angular momentum $L_{z}=$ $\left[G M a\left(1-e^{2}\right)\right]^{1 / 2}$, while $\Delta E_{*}$ and $\Delta L_{z *}$ are the corresponding changes for the interacting star. The quantities $B$ and $C$ are of order unity and can be derived by three-body scattering experiments that treat the star-binary encounters one at a time (Hut \& Bahcall 1983; Q96). For each encounter, one solves nine coupled, secondorder differential equations supplied by 18 initial conditions. The initial conditions define a point in a nine-dimensional parameter space represented by the mass ratio $q=M_{2} / M_{1}$ of the binary, its eccentricity $e$, the mass of the incoming field star, its asymptotic initial speed $v$, its impact parameter at infinity $b$, and four angles describing the initial direction of the impact, its initial orientation, and the initial binary phase. A significant star-binary energy exchange (i.e., characterized by a dimensionless energy change $C>$ 1) occurs only for $v<V_{c}\left(M_{2} / M\right)^{1 / 2}$, where $V_{c}=(G M / a)^{1 / 2}$ is the binary orbital velocity (the relative velocity of the two holes if the binary is circular; see, e.g., Saslaw et al. 1974; Mikkola \& Valtonen 1992).

A set of 24 scattering experiments was performed for different binary mass ratios and initial eccentricities, each run tracking the orbital evolution of $4 \times 10^{6}$ stars. The binary evolution in an isotropic stellar background of density $\rho$ and one-dimensional velocity dispersion $\sigma$ at infinity is determined by three dimensionless quantities (Q96): the hardening rate

$$
H=\frac{\sigma}{G \rho} \frac{d}{d t}\left(\frac{1}{a}\right)
$$

the mass ejection rate $\left(M_{\mathrm{ej}}\right.$ is the stellar mass ejected by the binary)

$$
J=\frac{1}{M} \frac{d M_{\mathrm{ej}}}{d \ln (1 / a)}
$$

and the eccentricity growth rate

$$
K=\frac{d e}{d \ln (1 / a)}
$$

The hardening rate $H$ is approximately constant for separations smaller than the "hardening radius" (Q96),

$$
a<a_{h}=\frac{G M_{2}}{4 \sigma^{2}} .
$$

The binary is assumed to be embedded in a bulge of mass $M_{B}$, radius $R_{B}$, and stellar density profile approximated by a singular isothermal sphere (SIS). Stars are counted as "ejected" from the bulge if, after three-body scattering, their velocity $V$ far away from the binary is greater than the escape velocity from the radius of influence of the binary, $r_{\text {inf }} \equiv G M /\left(2 \sigma^{2}\right)$. The SIS potential is $\phi(r)=-2 \sigma^{2}\left[\ln \left(G M_{B} / 2 \sigma^{2} r\right)+1\right]$ (for $\left.r<R_{B}=G M_{B} / 2 \sigma^{2}\right)$, and the escape speed from $r_{\text {inf }}$ is then

$$
v_{\mathrm{esc}} \equiv \sqrt{-2 \phi\left(r_{\mathrm{inf}}\right)}=2 \sigma \sqrt{\left[\ln \left(M_{B} / M\right)+1\right]}=5.5 \sigma,
$$

where the second equality comes from the adopted bulge-black hole mass relation $M=0.0014 M_{B}$ (Haring \& Rix 2004). Stars that do not acquire a kick velocity large enough to escape the host bulge, i.e., with

$$
V<v_{\text {ret }}=\sqrt{2 \phi\left(R_{B}\right)-2 \phi\left(r_{\mathrm{inf}}\right)}=2 \sigma \sqrt{\ln \left(\frac{M_{B}}{M}\right)}=5.1 \sigma
$$

are allowed multiple interactions with the binary. They can return to the central regions on small-impact parameter orbits and undergo a second superelastic scattering ("secondary slingshots"). Secondary scatterings are not allowed for stars ejected with $5.1 \sigma \lesssim V \lesssim 5.5 \sigma$, since even a small deviation from sphericity of 


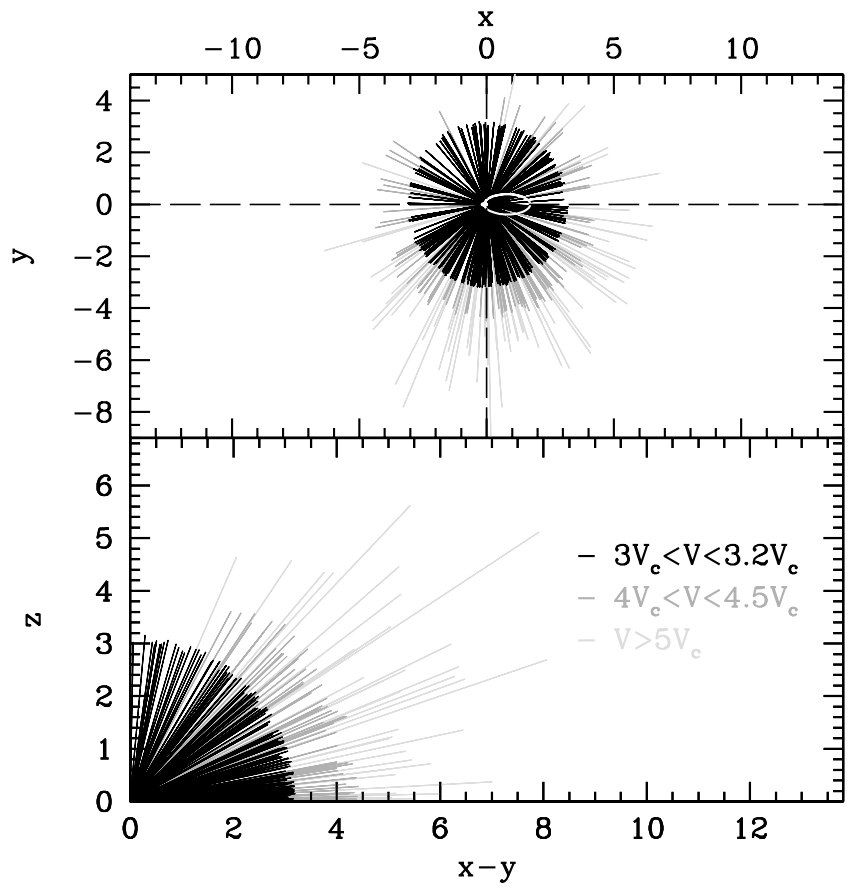

Fig. 1.- Velocity diagram of scattered stars in three different speed ranges: $3 V_{c}<V<3.2 V_{c}$ (black vectors), $4 V_{c}<V<4.5 V_{c}$ (gray vectors), and $V>$ $5 V_{c}$ (light gray vectors). Top: Longitude diagram of scattered stars. Each vector length is proportional to the modulus of the star's total velocity (not to the velocity projected into the $x-y$ plane). The ellipse shows the counterclockwise orbit of the lighter black hole of the binary. Bottom: Latitude diagram of scattered stars. [See the electronic edition of the Journal for a color version of this figure.]

the galaxy gravitational potential would make them miss the shrinking MBHB on their return to the center. Note that even if they were able to undergo another interaction with the binary, such stars would not contribute significantly to binary hardening as long as the condition $V>V_{c}\left(M_{2} / M\right)^{1 / 2}=2 \sigma\left(a_{h} / a\right)^{1 / 2}$ is satisfied, since in this case the star-binary energy exchange would be negligible.

As shown in Figure 1 and discussed in detail in Paper I, threebody interactions create a subpopulation of HVSs on nearly radial orbits, with a spatial distribution that is initially highly flattened in the inspiral plane of the binary, but becomes more isotropic with decreasing binary separation. The degree of anisotropy is smaller for unequal mass binaries and larger for stars with higher kick velocities. Eccentric MBHBs produce a more prominent tail of high-velocity stars and break axisymmetry, ejecting HVSs along a broad jet perpendicular to the semimajor axis. The jet two-sidedness decreases with increasing binary mass ratio, while the jet opening angle increases with decreasing kick velocity and orbital separation.

\subsection{Loss Cone Time Evolution}

In the absence of loss cone refilling by two-body relaxation or other processes, the supply of stars that can interact with the black hole pair is limited. Analytic expressions for nonequilibrium loss cone dynamics based on the evolution of the stellar distribution function as a result of repeated ejections have been given in MM03. Here we adopt a hybrid approach instead, combining the results of scattering experiments with an extension of MM03's study.

\subsubsection{Stellar Content}

The stellar content of the loss cone can be estimated from simple geometric considerations. When the MBHB separation is $a \lesssim a_{h}$, only a small fraction of bulge stars have low-angular momentum trajectories with pericenter distance $r_{p}<a$. In a spherical velocity distribution, the fraction of trajectories originating at $r$ and crossing a sphere of radius $a<r$ around the center is

$$
\Theta(r)=\left[1-\sqrt{1-\left(\frac{a}{r}\right)^{2}}\right] .
$$

The stellar mass within the geometric loss cone is then

$$
M_{*}=\int_{0}^{a} 4 \pi r^{2} \rho(r) d r+\int_{a}^{R_{B}} 4 \pi r^{2} \rho(r) \Theta(r) d r
$$

For a SIS $\rho(r)=\sigma^{2} /\left(2 \pi G r^{2}\right)$, and equation (10) is readily integrated to yield in the limit $a \ll R_{B}$

$$
M_{*} \simeq \frac{\pi \sigma^{2}}{G} a=\frac{\pi}{4}\left(\frac{a}{a_{h}}\right) M_{2} .
$$

The above scheme is oversimplified, as it assumes stellar trajectories to be straight lines. The gravitational field of the stellar mass distribution increases the net number of distant stars with pericenter distances $r_{p}<a$. Consider a star at distance $r>a$ from the binary moving with random velocity $v$. For a SIS, conservation of energy gives

$$
v^{2}=v_{p}^{2}+4 \sigma^{2} \ln \left(r_{p} / r\right)
$$

where $v_{p}$ is the star's velocity at pericenter. If $b$ is the impact parameter at distance $r$, angular momentum conservation yields

$$
b^{2}=r_{p}^{2}\left[1+4\left(\sigma^{2} / v^{2}\right) \ln \left(r / r_{p}\right)\right] .
$$

The second integral on the right-hand side of equation (10) can then be rewritten as

$$
\begin{aligned}
& \int_{a}^{R_{B}} 4 \pi r^{2} \rho(r) \Theta(r)\left\{\int_{0}^{\infty} 4 \pi v^{2} f(v)\right. \\
& \left.\times\left[1+4\left(\frac{\sigma^{2}}{v^{2}}\right) \ln \left(\frac{r}{a}\right)\right] d v\right\} d r,
\end{aligned}
$$

where $f(v)$ is the stellar velocity distribution. For a Maxwellian, the above equation can be simplified by setting $v=\langle v\rangle=\sqrt{3} \sigma$ in equation (13); one can then define a $\Theta$-factor that includes gravitational focusing

$$
\Theta(r) \rightarrow \Theta(r) \simeq 3.1\left[1-\sqrt{1-\left(\frac{a}{r}\right)^{2}}\right]\left[1+\frac{4}{3} \ln \left(\frac{r}{a}\right)\right] .
$$

Numerical integration of equation (10) finally yields for the stellar mass in the loss cone

$$
M_{*} \simeq \frac{8.2 \sigma^{2}}{G} a \simeq 2\left(\frac{a}{a_{h}}\right) M_{2} .
$$

Note that a fraction $\sim 0.5 M_{2}$ of the mass contained in the loss cone when the binary becomes hard $\left(a=a_{h}, M_{*} \simeq 2 M_{2}\right)$ lies within $a_{h}$. Let $t=0$ be the time at which the binary separation is $a=a_{h}$. The number flux of stars into the geometric loss cone, 
i.e., the flux of stars with $r>a_{h}$ at $t=0$ that interact with the binary at a later time $t$, is

$$
\mathcal{F} \simeq \frac{2 \sqrt{3} \sigma^{3}}{G m_{*} \pi a_{h}^{2}} \Theta
$$

where $\Theta=\Theta\left(\sqrt{3} \sigma t+a_{h}\right)$.

\subsubsection{Energy Exchange}

Let us denote with $\mathcal{E}$ the total binding energy of the MBHB, $\mathcal{E}=G M_{1} M_{2} / 2 a$. The total energy transfer rate from the binary to stars in the loss cone can be written as

$$
\frac{d \mathcal{E}}{d t} \simeq \frac{\Delta E_{*}(a) M_{*}(a)}{t_{\mathrm{ch}}},
$$

where $t_{\mathrm{ch}}$ is a characteristic interaction timescale and $\Delta E_{*}(a) \sim$ $G \mu / a$ is the characteristic specific energy gain of stars as a consequence of the gravitational slingshot. MM03 have written equation (18) in the case of returning stars, i.e., kicked stars that do not escape the host bulge and can have a secondary superelastic interaction with the MBHB. Returning stars have energy $E(a) \sim \phi\left(r_{\text {inf }}\right)+$ $\Delta E_{*}(a)$, and their interaction timescale $t_{\mathrm{ch}}$ can be identified with the typical radial period of stars in an SIS potential,

$$
\begin{aligned}
t_{\mathrm{ch}} \sim P(E) & =P(0) \exp \left(E / 2 \sigma^{2}\right) \\
& =P(0)\left(\frac{M}{M_{B}}\right) \exp \left[\left(\frac{2 C}{1+q}\right)\left(\frac{a_{h}}{a}\right)-1\right],
\end{aligned}
$$

where $P(0)=\sqrt{\pi} G M_{B} / 2 \sigma^{3}$ is of order the bulge crossing time and the average dimensionless energy change $C$ is of order unity and nearly independent of $a$ for $a<a_{h}$. As noted by MM03, in this case $M_{*}(a) \Delta E_{*}(a) \propto a^{1} a^{-1} \sim$ const. From equation (16) simple calculations lead to

$$
\frac{a_{h}}{a} \simeq \frac{a_{h}}{a_{1}}+\frac{1+q}{2 C} \ln \left[1+8 C^{2} \frac{q}{(1+q)^{2}} \frac{\left(t-t_{1}\right)}{P_{1}}\right],
$$

where $a_{1}$ is the binary separation at time $t=t_{1}$ when secondary slingshots start and $P_{1}$ is the period of stars with energy $E\left(a_{1}\right)$.

MM03's analysis can be expanded to account for the effect of three-body scatterings when the MBHB first becomes hard at separation $a=a_{h}$. Stars with $r<a_{h}$ at $t=0$ will interact with the binary within a timescale $t_{\mathrm{ch}} \sim a_{h} /(\sqrt{3} \sigma)$. Substitution of $t_{\mathrm{ch}}$ in equation (18), followed by simple algebra, leads to the expression

$$
\frac{a_{h}}{a}=1+C\left(\frac{q}{1+q}\right)\left(\frac{\sqrt{3} \sigma}{a_{h}}\right) t .
$$

A further contribution to the shrinking is associated with stars having $r>a_{h}$ at $t=0$ that are bound to enter the loss cone at later times. This population has total mass $\sim 1.5 \mathrm{M}_{2}$, and its contribution to the orbital decay is given by

$$
\frac{d \mathcal{E}}{d t} \simeq \Delta E_{*}(a) m_{*} \mathcal{F} \pi a^{2} .
$$

A straightforward substitution gives

$$
\frac{d}{d t}\left(\frac{a_{h}}{a}\right)=C\left(\frac{q}{1+q}\right)\left(\frac{\sqrt{3} \sigma}{a}\right) \Theta\left(\sqrt{3} \sigma t+a_{h}\right) .
$$

The above equation holds for a bulge crossing time $t<R_{B} / \sqrt{3} \sigma$ and must be solved numerically.

\subsubsection{Orbital Decay}

The simple analytical formulation described above can be refined using results from our scattering experiments (Paper I). This allows us to follow at the same time both the orbital decay of the binary and the evolution of the distribution function of interacting stars. The procedure is the following. We first isolate, from the initial distribution of kicked stars, the new loss cone, i.e., the subset of stars returning to the center on orbits with $r_{p}<$ $a_{1}$, where $a_{1}$ is the binary separation at the end of the first interaction with the stellar background. Then we compute the hardening rate $H$ by averaging $H_{1}(v)$ (provided by our scattering experiments) over the velocity distribution function of such stars, which are allowed to interact with the binary for the timescale in equation (19), again averaged over the stellar velocity distribution. After each step, the velocity distribution function of returning stars is updated and so is the timescale of the following interaction. We iterate the process until the loss cone is emptied. Convergence to the final stalling separation is usually obtained after $\gtrsim 4$ iterations. The mathematical details of the numerical procedure are given in the Appendix.

In order to specify the two parameters defining the SIS, the Haring \& Rix (2004) bulge-black hole mass relation was complemented by the $M-\sigma$ relation (Ferrarese \& Merritt 2000; Gebhardt et al. 2000) proposed by Tremaine et al. (2002),

$$
\sigma_{70}=0.84 M_{6}^{1 / 4},
$$

where $\sigma_{70}$ is the stellar velocity dispersion in units of $70 \mathrm{~km} \mathrm{~s}^{-1}$ and $M_{6}$ is the MBHB mass in units of $10^{6} M_{\odot}$. Using equation (24), the hardening radius can then be written as

$$
a_{h} \simeq 0.32 \mathrm{pc} M_{2,6}^{1 / 2} \sqrt{\frac{q}{1+q}},
$$

where $M_{2,6} \equiv M_{2} / 10^{6} M_{\odot}$. The binary separation as a function of time is shown in Figure 2, where it is also compared to the results of an $\mathrm{N}$-body simulation and to an analytical prescription, both presented in MM03 (their Fig. 6). The agreement with the simulation is fairly good. Our results, in terms of the final separation achieved as a function of $q$, are perfectly consistent with the stalling radii estimated by Merritt (2006).

Figure 2 shows how the rate of orbital decay declines after a few bulge crossing times $\sim P(0)=1.32 \times 10^{7} \mathrm{yr} M_{6}^{1 / 4}$ due to the decreasing supply of low-angular momentum stars from the outer regions of the bulge, once the stars in the central cusp have interacted with the binary. Note that equal-mass binaries shrink more than unequal binaries because of both the scaling of the stellar mass available for the interaction and the energy exchanged during a typical three-body encounter. It is easy to see that $d(1 / a) / d \ln t \propto q$. Eccentricity plays a marginal role in the orbital evolution of the MBHB. For a given $q$, the orbital shrinking is larger by at most $10 \%$ for highly eccentric binaries.

Figure 3 shows an example of the role of secondary slingshots on orbital shrinking for an equal-mass circular binary. The bottom panel clearly illustrates how successive interactions of stars returning on quasi-radial orbits can reduce the final binary separation by an extra factor of order 2 , i.e., $a_{f} \sim a_{1} / 2$. The progressive emptying of the loss cone is sketched in the top panel, where we plot the (differential) mass in stars approaching the binary with a given periastron. After the first interaction, only a few stars are 


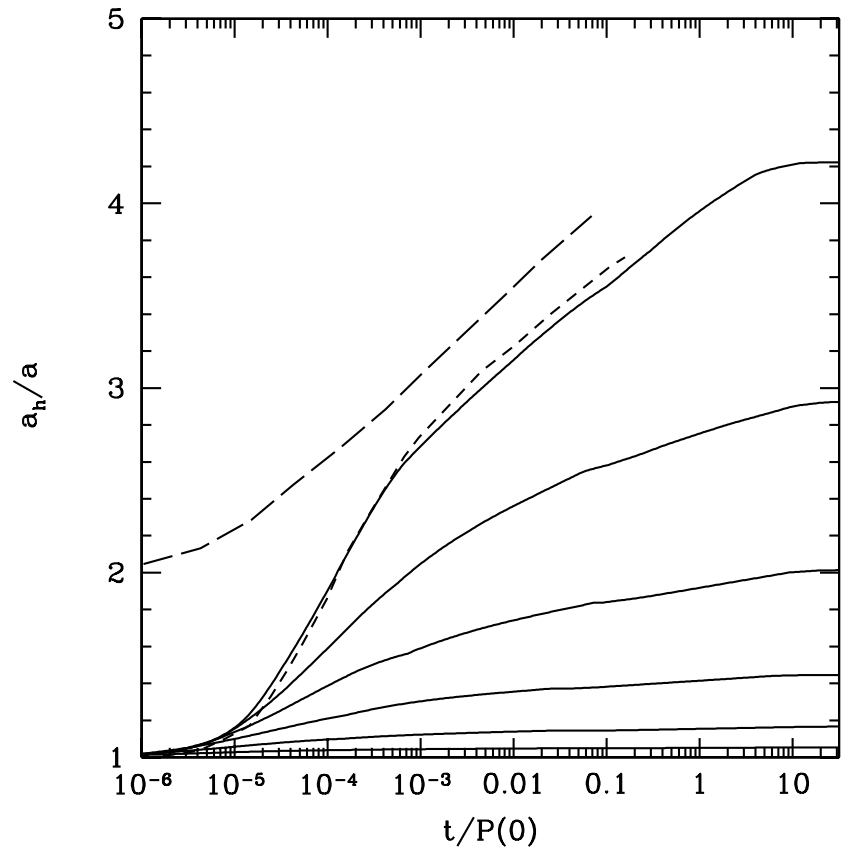

FIG. 2.-Decay of binary separation $a$ (in units of $a_{h}$ ) as a function of time [in units of the bulge crossing time $P(0)=1.32 \times 10^{7}$ yr $M_{6}^{1 / 4}$ for a SIS]. The solid lines, from bottom to top, are for $q=1 / 243,1 / 81,1 / 27,1 / 9,1 / 3$, and 1 . The $q=1$ case is compared to the analytical estimate (long-dashed line) of MM03 and to an $\mathrm{N}$-body simulation (short-dashed line) of MM03 performed with 18,000 stars initially in the loss cone, and the stellar potential is replaced by a smooth component to prevent relaxation. We set $M=0.0014 M_{B}$ and use the $M-\sigma$ relation (eq. [24]).

kicked out from the bulge. The loss cone, while substantially hotter, remains nearly full and only gets progressively depleted as secondary slingshots take place. Table 1 quantifies the role of returning stars for different values of the binary mass ratio $q$; returning stars can increase the shrinking of the MBHB by as much

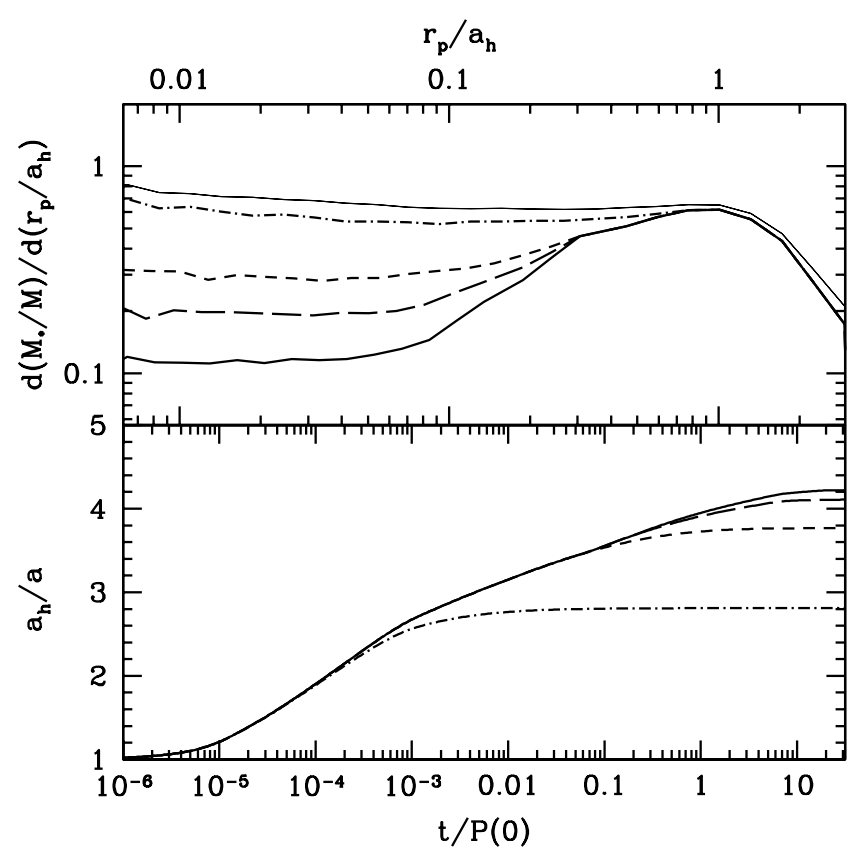

FIG. 3.-Top: Evolution of the loss cone population in terms of the (differential) stellar mass that approaches the binary with a given periastron $r_{p}$. The thin line shows the initial loss cone. The thick lines, from top to bottom, represent the loss cone population after the first, second, third, and fourth interactions. Bottom: Binary separation as a function of time. From bottom to top, the lines depict the shrinking associated with only the first one, two, three, and four interactions. An equal-mass, circular binary is assumed.
TABLE 1

Binary Hardening: The Impact of Returning Stars

\begin{tabular}{|c|c|c|}
\hline$q$ & $a_{h} / a_{1}$ & $a_{h} / a_{f}$ \\
\hline 1 & 2.81 & 4.41 \\
\hline $1 / 3$ & 2.19 & 3.07 \\
\hline $1 / 9$ & 1.64 & 2.09 \\
\hline $1 / 27$ & 1.29 & 1.49 \\
\hline $1 / 81$ & 1.12 & 1.19 \\
\hline $1 / 243$ & 1.04 & 1.06 \\
\hline
\end{tabular}

NoтE.-Binary shrinking factors, where $a_{h} / a_{1}$ is the binary shrinking after the first interaction only, while $a_{h} / a_{f}$ takes into account the subsequent reejections up to the fourth interaction.

as a factor of 2 and play a larger role for equal-mass binaries. This is because binary-to-star energy exchange is significant only for $V \lesssim \sqrt{q} V_{c}$ (see Paper I). After the first binary-star interaction, the stellar population is heated up and stars have on average $V \sim \sqrt{q} V_{c}$. In the case $q=1$, the binary shrinks by a significant factor, $V_{c}$ increases, and most of the returning stars have $V \lesssim V_{c}$ : the hardening process is still efficient. By contrast, when $q \ll 1$, $V_{c}$ does not increase appreciably after the first interaction, returning stars have $V \sim \sqrt{q} V_{c}$, and binary hardening stops.

\section{DISCUSSION}

Under the assumed criterion for stellar ejection, we can compute $M_{\mathrm{ej}}$, the mass of stars expelled with $V>v_{\mathrm{esc}}$. An example of the effects of the slingshot mechanism on the stellar population is shown for an equal-mass circular binary in Figure 4, where the initial $(t=0)$ velocity distribution of interacting stars is compared to the distribution after loss cone depletion. As already mentioned in $\S 2$, after the first interaction with the binary a large subset of kicked stars still lies in the loss cone of the

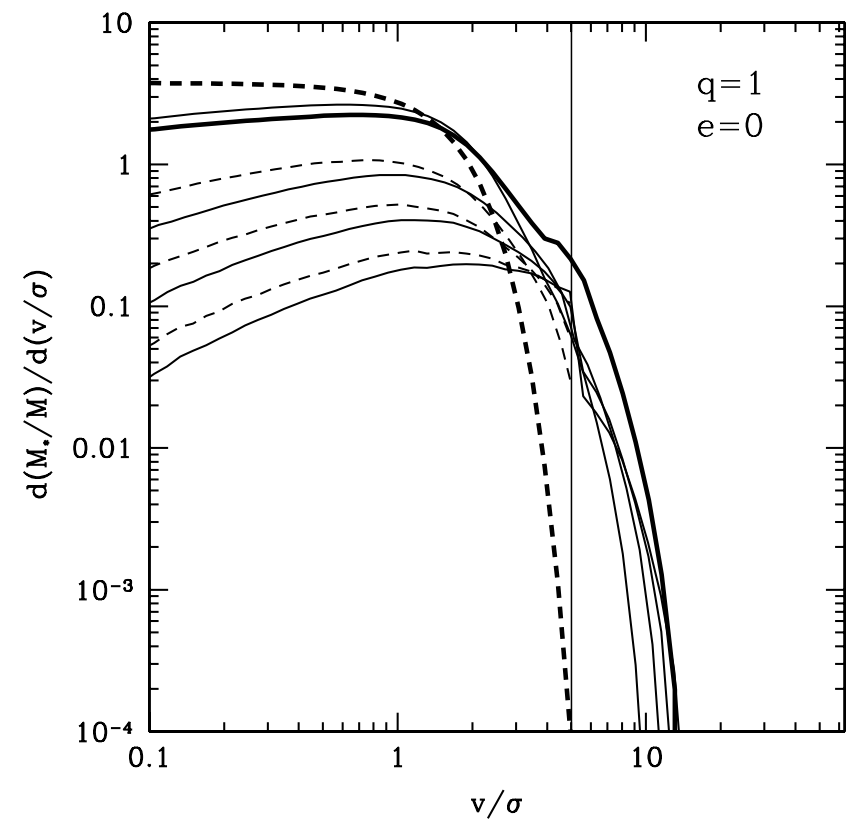

FIG. 4.- Stellar velocity distribution for an equal-mass, circular binary at different stages of binary hardening. The vertical line marks $v_{\text {ret }}=5.1 \sigma$ (we recall that $v_{\text {esc }}=5.5 \sigma$ ). The dashed lines, from top to bottom, represent the distribution of stars in the shrinking loss cone before the first, second, third, and fourth iterations. For clarity, the initial loss cone distribution is marked with a thicker line. The thin solid lines, from top to bottom, represent the distribution of stars that have received one, two, three, and four kicks. The thick solid line represents the final stellar velocity distribution after loss cone depletion is completed. 


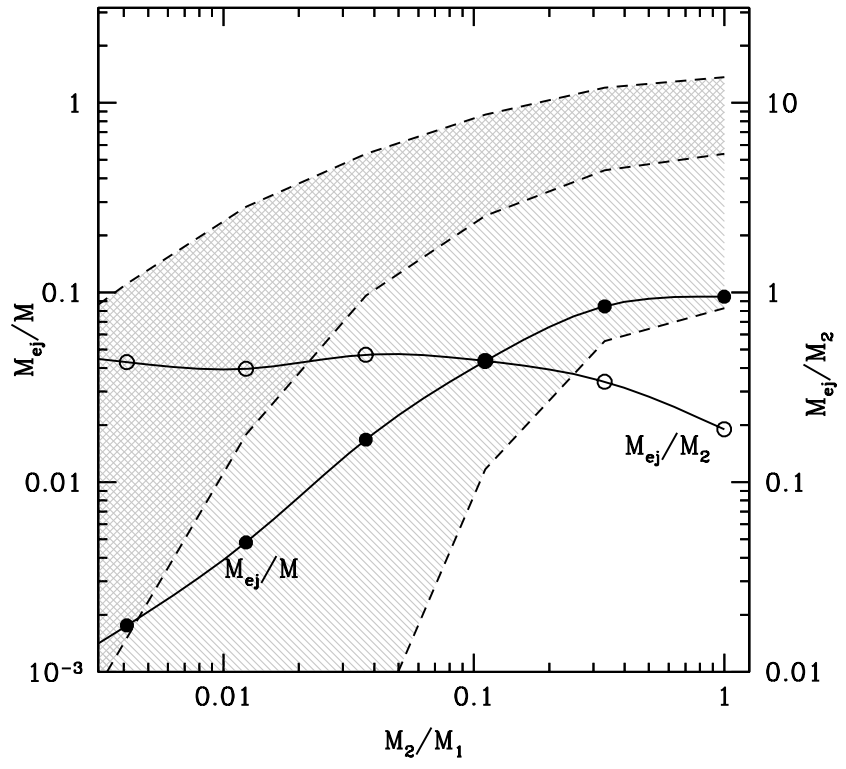

FIG. 5.-Ejected stellar mass $M_{\mathrm{ej}}$ normalized to the total binary mass $M$ (left scale, filled circles) and to the mass of the lighter binary member $M_{2}$ (right scale, open circles) as a function of binary mass ratio. The lines are polynomial interpolations. Note that the ratios $M_{\mathrm{ej}} / M$ and $M_{\mathrm{ej}} / M_{2}$ do not depend on the absolute value of $M$ and are nearly independent of $e$. Upper, dark shaded area: The mass (normalized to $M$ ) a $M=10^{6} M_{\odot}$ binary needs to eject to reach a final separation $a_{f}$ such that $t_{\mathrm{GW}}=1$ Gyr. Top and bottom boundaries assume $e=0$ and 0.9 , respectively. Lower, light shaded area: Same as the upper, dark shaded area, but for a $M=10^{9} M_{\odot}$ binary.

shrinking binary, has velocities $v<v_{\text {ret }}$, and is potentially available for further interactions. These are the stars we termed "returning." While scattering with the binary increases the stellar velocity thus reducing the energy exchanged in secondary interactions, it moves kicked stars on more radial orbits, thus reducing their impact parameter as well. Our calculations show that the highvelocity tail of the distribution depends on the MBHB eccentricity $e$, although the effect of changing $e$ is small for small values of $q$. In this case, fewer stars are kicked out compared to the case $q \lesssim 1$, but at higher velocities on average. In general, both a small mass ratio and a high eccentricity increase the tail of HVSs. Integrating the curves in Figure 4 over velocity gives the mass of interacting stars; this is $\simeq 2 M$ for the case shown $(q=1), \simeq 1.2 M$ for $q=$ $1 / 3$, and $\simeq 0.6 M$ for $q=1 / 27$ (all assuming $e=0$; we checked that eccentricity plays a negligible role).

Figure 5 depicts the ejected mass $M_{\mathrm{ej}}$ normalized to $M$ (left scale) and to $M_{2}$ (right scale) as a function of $q$. Our results show that $M_{\mathrm{ej}} / M \sim 0.5 \mu / M=0.5 q /(1+q)^{2}$, i.e., $M_{\mathrm{ej}} / M_{2} \sim 0.5 /(1+q)$, both ratios being independent of the total binary mass. The rate of stellar mass ejection is shown in Figure 6 as a function of time. A fraction $\lesssim 50 \%$ of the expelled stars is ejected in an initial burst lasting $\sim a_{h} / \sigma$, this fraction being smaller for smaller binary mass ratios. The burst is associated with the ejection of those stars already present within the geometric loss cone when the binary first becomes hard. Note that, for small $q$, mass ejection is already significant at $a \simeq a_{h}$, as in this case the binary orbital velocity is $V_{c} \gtrsim v_{\text {esc }}$ for $a \lesssim a_{h}$.

Is the amount of ejected mass sufficient to shrink the MBHB orbit down to the GW-dominated regime? To answer this question, we start defining the "final separation" $a_{f}$ as the separation reached by the binary before complete loss cone depletion, i.e., after a few bulge crossing times $\left(\sim 10^{7} \mathrm{yr}\right.$, weakly depending on binary mass as $\left.\propto M^{1 / 4}\right)$. We must then compare $a_{f}$ to the sep-

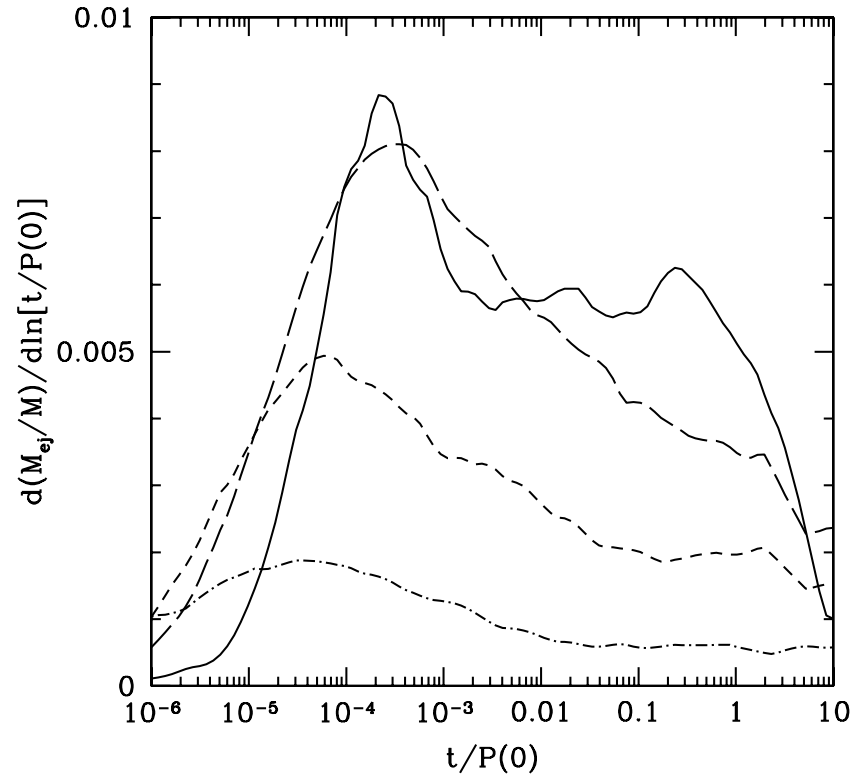

FIG. 6.-Ejected stellar mass per unit logarithmic time interval as a function of time. A circular binary is assumed. The solid, long-dashed, short-dashed, and dotdashed lines represent $q=1,1 / 3,1 / 9$, and $1 / 27$, respectively.

aration at which the orbital decay timescale from GW emission (Peters 1964),

$$
\begin{aligned}
t_{\mathrm{GW}} & =\frac{5 c^{5}}{256 G^{3}} \frac{a^{4}}{M_{1} M_{2} M F(e)} \\
& \approx 0.25 \mathrm{Gyr}\left(\frac{M M_{1} M_{2}}{10^{18.3} M_{\odot}^{3}}\right)^{-1} F(e)^{-1}\left(\frac{a}{0.001 \mathrm{pc}}\right)^{4},
\end{aligned}
$$

is shorter than, say, 1 Gyr. Here, to fourth order in $e$,

$$
F(e)=\left(1-e^{2}\right)^{-7 / 2}\left(1+\frac{73}{24} e^{2}+\frac{37}{96} e^{4}\right) .
$$

Inverting equation (26), one can define the separation $a_{\mathrm{GW}}$ at which the binary will coalesce in a given time $t$,

$$
\begin{aligned}
a_{\mathrm{GW}} & =\left[\frac{256 G^{3}}{5 c^{5}} t M_{1} M_{2} M F(e)\right]^{1 / 4} \\
& \approx 0.0014 \mathrm{pc}\left(\frac{M M_{1} M_{2}}{10^{18.3} M_{\odot}^{3}}\right)^{1 / 4} F(e)^{1 / 4} t_{9}^{1 / 4}
\end{aligned}
$$

where $t_{9} \equiv t / 1$ Gyr. Using equations (25) and (28), one finds that $a_{f} / a_{\mathrm{GW}} \propto M^{-1 / 4} q^{3 / 4}$ (see also MM03, eq. [90]), i.e., the more massive the binary and the smaller the binary mass ratio, the smaller the factor the binary must shrink to reach the GW emission regime. Eccentricity plays a double role. For a given binary mass and mass ratio, on one hand the hardening rate slightly increases with increasing eccentricity ( $\leqslant 20 \%$ from $e=0$ to 0.9 ), leading to a smaller $a_{f}$; on the other hand, from equations (27) and (28), $a_{\mathrm{GW}}$ is larger for larger $e$, thus reducing the $a_{h}-a_{\mathrm{GW}}$ gap. An important effect, included in our calculations, is that the eccentricity typically increases during the binary-star interaction, although the functional form of $F(e)$ is such that the effect is significant only for binaries with $e \gtrsim 0.6$ already at $a_{h}$. In other words, for MBHBs with initially low eccentricities, the increase 


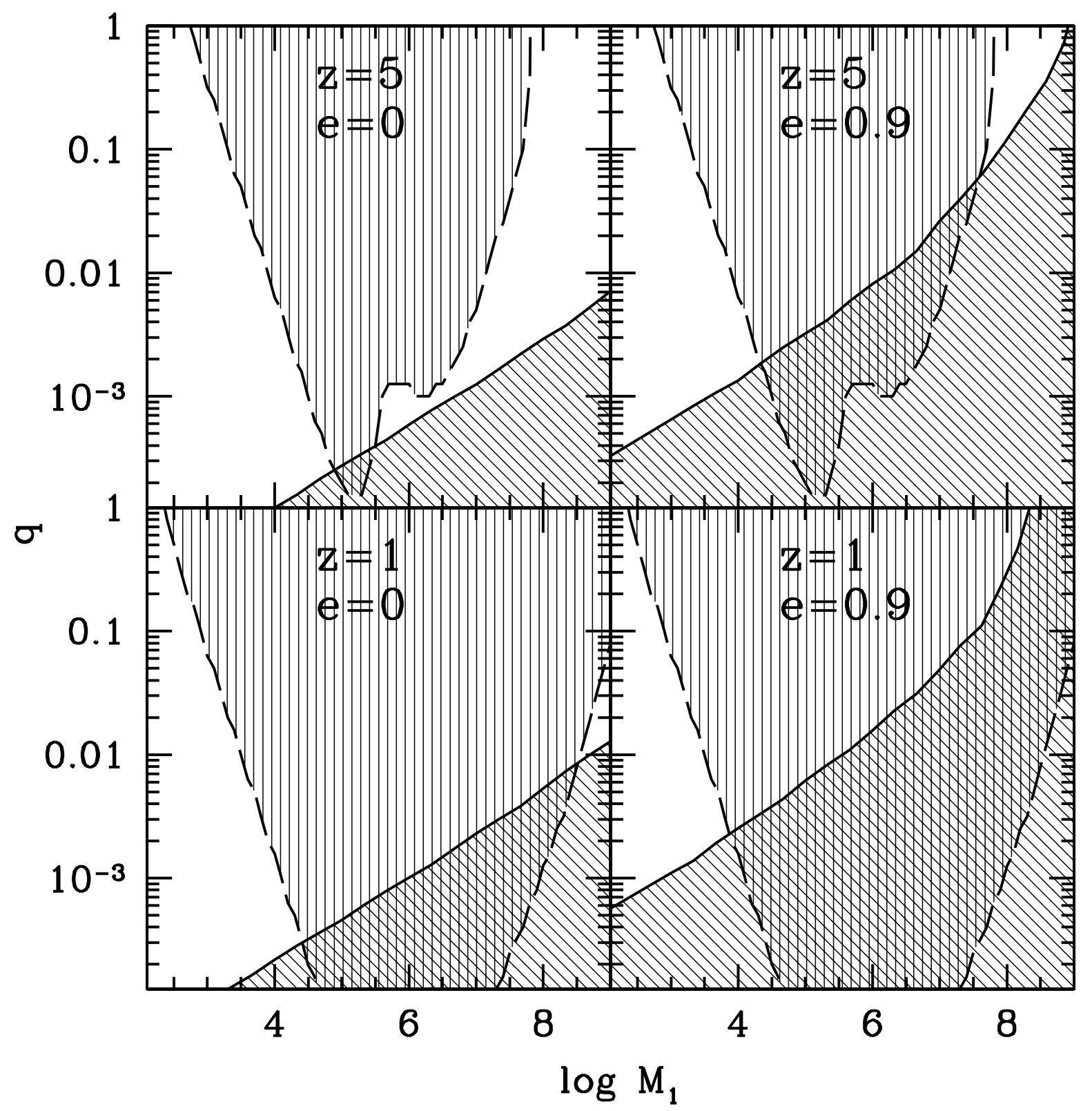

Fig. 7. - The $M_{1}-q$ plane. The vertical shaded area shows $L I S A$ potential targets with $\mathrm{S} / \mathrm{N}>5$. The diagonal shaded area in the bottom right corner marks binaries that will coalesce within a then Hubble time after loss cone depletion. In each panel, the assumed redshift and eccentricity of the MBHBs are labeled.

of $e$ during the gravitational slingshot affects only weakly the final $a_{f} / a_{\mathrm{GW}}$ ratio.

It is interesting to compare the total mass actually ejected prior to complete loss cone depletion with the stellar mass that must be expelled in order to reach a final orbital separation where $t_{\mathrm{GW}}\left(a_{f}\right)=1$ Gyr, i.e., where GW emission leads to coalescence within 1 Gyr. An example is shown in Figure 5. The shaded areas define such mass (in units of $M$ ) for a $10^{6}$ and a $10^{9} M_{\odot}$ MBHB, where the top boundary assumes $e=0$, and the bottom assumes $e=0.9$. Note how the $e=0.9, M=10^{6} M_{\odot}$ lower boundary practically coincides with the $e=0, M=10^{9} M_{\odot}$ upper one. The figure clearly shows how, even in the absence of other mechanisms driving orbital decay, pairs involving genuinely supermassive holes should not stall, while for lighter binaries both a small mass ratio and a large eccentricity are probably required for coalescence to take place.

Using our hybrid model, we can also sample the $\left(M_{1}, q, e\right)$ three-dimensional space, compute the separation $a_{f}$ and the ec- centricity $e$ at $a_{f}$, then fold the calculated values of $a_{f}$ and $e$ into equation (26), and finally compare $t_{\mathrm{GW}}$ to the Hubble time at two reference redshifts, $z=1$ and 5. In Figure 7 binaries that will coalesce within a then Hubble time after loss cone depletion populate the diagonally shaded area in the $M_{1}-q$ plane, while the vertically shaded area marks MBHBs that, if driven to coalescence by $z=1$ or 5 , would be resolved by LISA with a signal-to-noise ratio $\mathrm{S} / \mathrm{N}>5$ (see Sesana et al. 2005, and references therein for details). The region of overlap selects unequal mass, highly eccentric MBHBs with $M \gtrsim 10^{5}$ that can shrink down to the $\mathrm{GW}$ emission regime in less than a Hubble time and that are "safe" targets for LISA even in the pessimistic case, treated here, of stellar slingshots + loss cone depletion with no refilling.

It is important to remark at this stage that our calculations are meant to define a minimal model for the evolution of MBHBs and that several other mechanisms may help the orbital decay and widen the range of potential LISA targets. First, we have assumed all the stars in the loss cone to be unbound to the MBHs. 
In a realistic case, each $\mathrm{MBH}$ will bind stars inside its radius of influence $r_{\text {inf }}$; the star binding energy can be extracted by slingshot, hence enhancing binary hardening. This effect is not expected to be important for equal-mass binaries, as in this case $a_{h} \sim r_{\text {inf }}$ and only a small fraction of interacting stars will be bound to the binary. Indeed, our results match well the numerical simulations of MM03 (Fig. 2). For lower mass ratios, however, it is $a_{h} \ll r_{\text {inf }}$, and most stars in the loss cone are actually bound to the binary. A forthcoming paper will be devoted to an analysis of three-body scattering experiments for a MBHB with bound stars, providing a more realistic model for the case $q \ll 1$.

Second, even in spherical stellar bulges, loss cone refilling due to two-body relaxation ( Yu 2002; MM03) and the wandering of the black hole pair in the nucleus (Quinlan \& Hernquist 1997; Chatterjee et al. 2003) could both increase the amount of stellar mass interacting with the MBHB. The two-body relaxation timescale is such that loss cone refilling is probably important for $M \lesssim 10^{6} M_{\odot}$. The Brownian motion timescale is of the order of 15 Gyr for a $10^{6} M_{\odot}$ binary and scales as $M^{5 / 4}$. It is then likely that the two effects considered here may affect orbital decay only for light binaries, helping them to cover the residual gap between $a_{f}$ and $a_{\mathrm{GW}}$ and leading light binaries to coalesce within a then Hubble time even at high redshifts. On the other hand, their contribution to the shrinking of supermassive binaries with $M \gtrsim$ $10^{6} M_{\odot}$ is probably negligible.

If the stellar bulge is not spherical, but axisymmetric, stars on highly eccentric orbits are typically centrophilic (Touma \& Tremaine 1997; Magorrian \& Tremaine 1999). In this case, the loss cone is substituted by a "loss wedge" (see Yu [2002] for a detailed discussion). The stellar content of such a wedge is larger than that of the corresponding loss cone and depends on the degree of flattening $\epsilon$ of the stellar distribution. Typically, for a galaxy with $\epsilon=0.3$, the stellar content of the loss wedge is an order of magnitude larger than the stellar content of the loss cone if the bulge is spherical ( Yu 2002). Note that Faber et al. (1997) estimate an average $\epsilon=0.36$ for a sample of galaxies, leading to the conclusion that the hardening of a MBHB in such a potential might be much faster than our "spherical" estimate. We also recall that triaxial potentials drive many bulge stars on chaotic orbits, many of them centrophilic. One then expects an increase in the number of interacting stars similar to that produced by axisymmetric potentials (Merritt \& Poon 2004; Berczik et al. 2006).

Finally, MBHB orbital evolution can also, at least partially, be driven by drag in a gaseous nuclear disk. The role of gas is basically twofold. On $100 \mathrm{pc}$ scales, the disk drastically increases dynamical friction, reducing the timescale on which MBHs can reach the center of the bulge (Escala et al. 2004; Dotti et al. 2006). On parsec scales, torques induced by the disk can drive the binary to decay on a timescale of order the gas accretion time (Ivanov et al. 1999; Armitage \& Natarajan 2002). It is important to point out that the interaction with a gaseous disk typically circularizes the binary orbit (Dotti et al. 2006), hence maximizing the $a_{h}-a_{\mathrm{GW}}$ gap. If this is the case, the slingshot-driven coalescence would be more difficult to achieve.

Support for this work was provided by the Italian MIUR grant PRIN 2004 (A. S. and F. H.) and by NASA grants NAG 0511513 and NNG 04-GK85G (P. M.). P. M. also acknowledges support from the Alexander von Humboldt Foundation.

\section{APPENDIX}

\section{NUMERICAL INTEGRATION OF THE MBHB ORBITAL DECAY}

The average hardening rate for a Maxwellian stellar velocity distribution $f(v, \sigma)=\left(2 \pi \sigma^{2}\right)^{-3 / 2} \exp \left(-v^{2} / 2 \sigma^{2}\right)$ is given by

$$
H(\sigma) \equiv \int_{0}^{\infty} f(v, \sigma) \frac{\sigma}{v} H_{1}(v) 4 \pi v^{2} d v
$$

where

$$
H_{1}(v) \equiv 8 \pi \int_{0}^{\infty}\langle C\rangle x d x
$$

is the dimensionless hardening rate if all stars have the same velocity $v, x \equiv b /\left(2 G M a / v^{2}\right)^{1 / 2}$ is the dimensionless impact parameter, and the energy exchange $\langle C\rangle$ is averaged over the orbital angular variables (Paper I; Q96). An expression analogous to equation (A1) relates the thermally averaged eccentricity growth rate $K(\sigma)$ to $K_{1}(v)$,

$$
K_{1}(v) \equiv \frac{\left(1-e^{2}\right)}{2 e} \frac{\int_{0}^{\infty}\langle B-C\rangle x d x}{\int_{0}^{\infty}\langle C\rangle x d x},
$$

where $\langle B-C\rangle$ is the mean angular momentum minus energy exchange. For a binary with given mass ratio and eccentricity, the quantities $C$ and $B$, and thus $H_{1}$ and $K_{1}$, are functions of only the ratio $v / V_{c} \propto v \sqrt{a}$, where $V_{c}$ is the binary circular velocity. Given an incoming velocity $v$, we record the bivariate distribution $h_{1}\left(V, b^{\prime} \mid v\right)$ of stars with ejection speeds in the interval $[V, V+d V]$, leaving the binary with an "exit" impact parameter in the interval $\left[b^{\prime}, b^{\prime}+d b^{\prime}\right]$. The distribution function is normalized so that

$$
\int_{0}^{\infty} \int_{0}^{\infty} h_{1}\left(V, b^{\prime} \mid v\right) d V d b^{\prime}=1 .
$$

The subscript " 1 " indicates that the scattering experiments are performed for a binary at separation $a=1$.

The interaction of the MBHB with stars in the loss cone is assumed to take place in discrete steps. The binary first interacts with a given population of stars and shrinks accordingly. We then isolate the returning subpopulation, which becomes the input for the next 
step, and so on. Consider the binary at separation $a_{i}$, interacting with a stellar population of mass $M_{*, i}$ and (normalized) velocity distribution $f_{i}(v)$. The orbit decays according to the differential equation

$$
\frac{d}{d t}\left(\frac{1}{a}\right)=\frac{G \rho}{\langle v\rangle} H(a),
$$

where

$$
H(a)=\int_{0}^{\infty} f_{i}(v) \frac{\langle v\rangle}{v} H_{1}(v \sqrt{a}) d v
$$

and $\rho$ is the stellar density. Straightforward integration of equation (A5) gives

$$
t(a)=\frac{\langle v\rangle}{G \rho} \int_{a}^{a_{i}} \frac{d a^{\prime}}{a^{\prime 2} H\left(a^{\prime}\right)},
$$

the time the orbit needed to shrink from $a_{i}$ to $a$. The solution above is not physically meaningful, as the time variable involved depends on the particular value assumed for $\rho$. Stated more directly, to solve equation (A5) we need to set a realistic pace at which star-binary interactions occur. This can be done in two steps.

First, we write the stellar mass $M_{*}$ that will interact with the binary in a given time $t$ as

$$
M_{*}(t)=k_{*} t,
$$

where

$$
k_{*} \equiv \int_{0}^{\infty} f_{i}(v) \pi b_{\max }^{2}(v) \rho v d v
$$

and the maximum allowed impact parameter is

$$
b_{\max }^{2}(v)=a_{i}^{2}\left(1+\frac{2 G M}{a_{i} v^{2}}\right) .
$$

Equation (A8) is valid as long as $M_{*}(t) \leq M_{*, i}$. Now we simply write $t=M_{*} / k_{*}$ and substitute into equation (A7), which now gives $M_{*}(a)$, the stellar mass interacting with the binary as the orbit shrinks from $a_{i}$ to $a$. Note that in $M_{*}(a)$ the term $\rho$ cancels out, i.e., the interacting mass is independent of any preassigned value for the stellar density. We can now numerically invert the equation for $M_{*}(a)$, obtaining $a\left(M_{*}\right)$, i.e., the binary separation as a function of the interacting mass. We define the final separation $a_{f} \equiv a\left(M_{*, i}\right)$. The very same procedure can be applied for the evolution of the binary eccentricity $e$, resulting in a function describing $e$ as a function of the interacting mass, $e=e\left(M_{*}\right)$.

We now need to relate $M_{*}$ to physical time. The stellar mass interacting with the binary per unit time is

$$
\frac{d M_{*}}{d t}=\frac{d M_{*}}{d v} \frac{d v}{d t}=f_{i}(v) M_{*, i} \frac{d v}{d t},
$$

where the term $d v / d t$ can be computed considering the typical interaction time for stars with velocity $v$ in a SIS potential, i.e., $t(v)=$ $P(0) \exp \left[\left(v^{2}-v_{\text {ret }}^{2}\right) / 4 \sigma^{2}\right]$. Straightforward algebra yields $d v / d t$ as a function of $t$. Equation (A11) can be then integrated, and the resulting $M_{*}(t)$ finally substituted into $a\left(M_{*}\right)$ to give the time evolution of the binary separation $a(t)$.

We can now compute the distribution of stars that, after the interaction, have velocities $V<v_{\text {ret }}$ and are then available for a further encounter with the MBHB. The bivariate distribution $h_{a}\left(V, b^{\prime} \mid v\right)$ can be extracted from the $h_{1}\left(V, b^{\prime} \mid v\right)$ distributions recorded in the scattering experiments. In the three-body problem integration, the binary mass and separation are taken as unity. This setup allows us to rescale the velocities and trajectories of kicked stars for any given physical value of the binary mass and separation. It can be shown that

$$
h_{a}\left(V, b^{\prime} \mid v\right)=\frac{1}{\sqrt{a}} h_{1}\left(\frac{V}{\sqrt{a}}, b^{\prime} a \mid v \sqrt{a}\right),
$$

where the prefactor $1 / \sqrt{a}$ normalizes the distribution according to equation (A4). The normalized distribution of scattered stars must be averaged over the input velocity $v$ and the separation $a$ and can be written as

$$
h\left(V, b^{\prime}\right)=\frac{\int_{a_{i}}^{a_{f}} \int_{0}^{\infty} f_{i}(a, v)\left(d M_{*} / d a\right) h_{a}\left(V, b^{\prime} \mid v\right) d v d a}{\int_{0}^{\infty} \int_{0}^{\infty} \int_{a_{i}}^{a_{f}} \int_{0}^{\infty} f_{i}(a, v)\left(d M_{*} / d a\right) h_{a}\left(V, b^{\prime} \mid v\right) d v d a d V d b^{\prime}},
$$


where the distribution of stars with velocity between $v$ and $v+d v$ that are going to approach the binary within a distance $<a$ is

$$
f_{i}(v, a)=\frac{f_{i}(v) \pi b^{2}(v, a)}{\int f_{i}(v) \pi b_{\max }^{2}(v) d v}
$$

where

$$
b^{2}(v, a)=a^{2}\left(1+\frac{2 G M}{a v^{2}}\right)
$$

and $b_{\max }(v)$ is given by equation (A10). The change of the interacting mass with binary separation $d M_{*} / d a$ is obtained by differentiation of the function $M_{*}(a)$, obtained above.

Finally, the (normalized) velocity distribution of returning stars $f_{r}(v)$ can be computed as

$$
f_{r}(v)=\frac{\int_{0}^{a_{f}} h\left(V, b^{\prime}\right) d b^{\prime}}{\int_{0}^{v_{\text {ret }}} \int_{0}^{a_{f}} h\left(V, b^{\prime}\right) d b^{\prime} d V}
$$

and the mass available for the subsequent interaction $M_{*, r}$ is given by

$$
M_{*, r}=M_{*, i} \frac{\int_{0}^{v_{\mathrm{ret}}} \int_{0}^{b\left(v, a_{f}\right)} h\left(V, b^{\prime}\right) d b^{\prime} d V}{\int_{0}^{\infty} \int_{0}^{\infty} h\left(V, b^{\prime}\right) d b^{\prime} d V} .
$$

The numerical procedure can be then iterated, considering the binary at a starting separation $a_{i}=a_{f}$, interacting with a stellar population whose (normalized) velocity distribution is $f_{i}(v)=f_{r}(v)$, allowing a total mass of interacting stars $M_{*, i}=M_{*, r}$. For the first interaction only, we assume that the stars already within the binary separation interact on a timescale $\sim a_{h} / \sigma$, along the lines discussed in $\S$ 2.2.2.

Aarseth, S. J. 2003, Ap\&SS, 285, 367

Armitage, P. J., \& Natarajan, P. 2002, ApJ, 567, L9

Begelman, M. C., Blandford, R. D., \& Rees, M. J. 1980, Nature, 287, 307

Berczik, P., Merritt, D., \& Spurzem, R. 2005, ApJ, 633, 680

Berczik, P., Merritt, D., Spurzem, R., \& Bischof, H.-P. 2006, ApJ, 642, L21

Chatterjee, P., Hernquist, L., \& Loeb, A. 2003, ApJ, 592, 32

Dotti, M., Colpi, M., \& Haardt, F. 2006, MNRAS, 367, 103

Escala, A., Larson, R. B., Coppi, P. S., \& Mardones, D. 2004, ApJ, 607, 765

Faber, S. M., et al. 1997, AJ, 114, 1771

Ferrarese, L., \& Merritt, D. 2000, ApJ, 539, L9

Gebhardt, K., et al. 2000, ApJ, 543, L5

Haring, N., \& Rix, H. W. 2004, ApJ, 604, L89

Hemsendorf, M., Sigurdsson, S., \& Spurzem, R. 2002, ApJ, 581, 1256

Hills, J. G. 1983, AJ, 88, 1269

Hut P., \& Bahcall, J. N. 1983, ApJ, 268, 319

Ivanov, P. B., Papaloizou, J. C. B., \& Polnarev, A. G. 1999, MNRAS, 307, 79

Kazantzidis, S., et al. 2005, ApJ, 623, L67

Komossa, S., Burwitz, V., Hasinger, G., Predehl, P., Kaastra, J. S., \& Ikebe, Y. 2003, ApJ, 582, L15

Magorrian, J., \& Tremaine, S. 1999, MNRAS, 309, 447

Magorrian, J., et al. 1998, AJ, 115, 2285
REFERENCES

Makino, J., \& Funato, Y. 2004, ApJ, 602, 93

Merritt, D. 2006, ApJ, 648, 976

Merritt, D., \& Poon, M. Y. 2004, ApJ, 606, 788

Mikkola, S., \& Valtonen, M. J. 1992, MNRAS, 259, 115

Milosavljevic, M., \& Merritt, D. 2001, ApJ, 563, 34 2003, ApJ, 596, 860 (MM03)

Peters, P. C. 1964, Phys. Rev. B, 136, 1224

Quinlan, G. D. 1996, NewA, 1, 35 (Q96)

Quinlan, G. D., \& Hernquist, L. 1997, NewA, 2, 533

Rodriguez, C., Taylor, G. B., Zavala, R. T., Peck, A. B., Pollack, L. K., \& Romani, R. W. 2006, ApJ, 646, 49

Saslaw, W. C., Valtonen, M. J., \& Aarseth, S. J. 1974, ApJ, 190, 253

Sesana, A., Haardt, F., \& Madau, P. 2006, ApJ, 651, 392 (Paper I)

Sesana, A., Haardt, F., Madau, P., \& Volonteri, M. 2005, ApJ, 623, 23

Sudou, H., Iguchi, S., Murata, Y., \& Taniguchi, Y. 2003, Science, 300, 1263

Touma, J., \& Tremaine, S. 1997, MNRAS, 292, 905

Tremaine, S., et al. 2002, ApJ, 574, 740

Volonteri, M., Haardt, F., \& Madau, P. 2003a, ApJ, 582, 559

Volonteri, M., Madau, P., \& Haardt, F. 2003b, ApJ, 593, 661

Yu, Q. 2002, MNRAS, 331, 935 